\title{
A Compact Quasi-Yagi Antenna with High Gain by Employing the Bent Arms and Split-Ring Resonators
}

\author{
Wen-Ying Zhou $\mathbb{D}^{1,2}$ Zhong-Lei Mei $\mathbb{D}^{3}{ }^{3}$ and Mai Lu $\mathbb{D}^{2}$ \\ ${ }^{1}$ School of Physical Science and Technology, Lanzhou University, Lanzhou Gansu 730000, China \\ ${ }^{2}$ Key Laboratory of Opt-Electronic Technology and Intelligent Control of Ministry of Education, Lanzhou Jiaotong University, \\ Lanzhou Gansu 730070, China \\ ${ }^{3}$ School of Information Science \& Engineering, Lanzhou University, Lanzhou Gansu 730000, China \\ Correspondence should be addressed to Zhong-Lei Mei; meizl@lzu.edu.cn
}

Received 14 November 2020; Revised 18 January 2021; Accepted 3 February 2021; Published 19 February 2021

Academic Editor: Muhammad Zubair

Copyright ( 2021 Wen-Ying Zhou et al. This is an open access article distributed under the Creative Commons Attribution License, which permits unrestricted use, distribution, and reproduction in any medium, provided the original work is properly cited.

\begin{abstract}
A compact quasi-Yagi antenna with bent arms and split-ring resonators (SRRs) is proposed. Compared with traditional quasiYagi antennas employing straight arms, the resonant frequency of the proposed antenna could be always consistent with its center frequency, and there is no obvious frequency shift under the process of its miniaturization. The SRRs are adopted in the proposed compact antenna for a high gain of $6.58 \mathrm{dBi}$. The reliability verification of the proposed antenna radiation characteristics is further experimentally proved with the prototype measurement. The proposed quasi-Yagi antenna has an adjustable compact structure and low frequency offset and could be used in the precise point-to-point wireless communication environment.
\end{abstract}

\section{Introduction}

As a classic directive antenna, Yagi-Uda has been widely used in many fields, such as RFID, WLAN, MIMO system, wireless energy harvesting, and IOT [1-5]. A traditional quasi-Yagi antenna consists of a dipole as a driven element, a reflector to cancel the back radiation, and one or more directors as parasitic elements to realize the end-fire radiation. The characteristics of the traditional quasi-Yagi antenna are decided by the proper amplitude and phase condition between its constituent elements. Therefore, a traditional quasi-Yagi antenna always has a large physical dimension for achieving a high gain at the S-band. For expanding the bandwidth and improving the gain of the Yagi antenna, different feed networks, baluns, exciters, and director structures design have been proposed [6-9].

As the electromagnetic environment becomes more complex, a miniaturized Yagi-Uda antenna is required to reduce electromagnetic interference and increase the flexibility of mobile terminals. However, in the process of YagiUda antenna miniaturization, the gain would decrease and the resonant frequency would deviate as well. Different compact Yagi antennas have been designed for different applications. In 2015 and 2017, a miniaturized quasi-Yagi antenna with fold dipole [10] and a compact printed YagiUda with a hairpin-shaped meandered driven element [11] were proposed for RFID. In 2018, a rectangular loop element was designed to realize the miniaturization of the quasi-Yagi antenna for constructing a multiwideband MIMO system [12]. In 2019, different filter elements were designed for obtaining the compact size of quasi-Yagi antennas in the S-band and C-band [13, 14]. In 2020, a compact quasi-Yagi antenna based on double monopole feed was proposed for broadband application [15], and a compact planar MoxonYagi composite antenna was designed for dual-band application [16].

Unlike the existing arc-shaped hairpin line [17] for gain enhancement and arrow-shaped line for broadening bandwidth [18], in this article, a compact quasi-Yagi antenna with bent-shaped arms of drivers and directors has been proposed, and there is no obvious frequency shift under the process of its miniaturization. Besides, the SRRs were 
adopted to obtain a high gain of the proposed antenna, and the specific influences of the bent arms and SRRs were further discussed in different compact structures of the proposed antenna. The final experimental results were satisfactory with simulation results. The flexibly adjustable characteristic of the proposed antenna, together with its low frequency deviation, is suitable to realize precise point-topoint communication in various fields, such as rail transit communication, WLAN, and industrial scientific and medical (ISM) in the frequency range of $2.4 \sim 2.48 \mathrm{GHz}$. If the frequency deviation range of an antenna exceeds $80 \mathrm{MHz}$ in abovementioned application fields, it will cause unpredictable electromagnetic compatibility problems in the whole communication system.

\section{Quasi-Yagi Antenna Design and Analysis}

2.1. Quasi-Yagi Antenna Design. By using the straight arms as the drivers and directors, a traditional quasi-Yagi is designed as a reference antenna, with the center frequency of $2.45 \mathrm{GHz}$, as shown in Figure 1. A dipole antenna is adopted as a driven element, and its two arms are printed on both sides of a dielectric substrate, respectively. An FR4 substrate slab is used, with a relative permittivity of 4.4 , thickness of $0.8 \mathrm{~mm}$, and dielectric loss tangent of 0.02 , which is used in all the antennas' design in this paper.

According to the length and width of the straight exciter and director's arm, a novel bent-arm antenna is proposed. An initial miniaturized quasi-Yagi based on the bent arm is shown in Figure 2. As the electric currents formed on bent arms are more suitable for wave propagation and radiation, the same radiation performance could be obtained when reducing the length of a quasi-Yagi antenna of size $(h)$ from $90 \mathrm{~mm}$ to $75 \mathrm{~mm}$.

\subsection{Characteristics Analysis}

2.2.1. Comparison of Radiation Performance. The reflection coefficients $\left(S_{11}\right)$ of quasi-Yagi antennas with straight and bent arms are simulated, respectively, by simulation software CST, and the comparison results are shown in Figure 3.

Figure 3 shows that both two antennas have good impedance matching at the same resonant frequency of $2.45 \mathrm{GHz}$ and obtain a bandwidth of $400 \mathrm{MHz}$.

Figure 4 shows that the end-fire radiation of both antennas is along the $y$-axis, and the gain of the quasi-Yagi antenna with bent arms is $6.13 \mathrm{dBi}$, which is slightly higher than that of the quasi-Yagi antenna with straight arms. Besides, there is a $17 \%$ reduction of the dielectric substrate length.

2.2.2. Comparison of Resonant Frequency Shift. By reducing the distance $\left(L_{3}\right)$ between directors and drivers and the distance $\left(L_{2}\right)$ between directors, the physical dimensions of the proposed quasi-Yagi antenna can be reduced correspondingly, when $L_{2}$ varies from $3 \mathrm{~mm}$ to $16 \mathrm{~mm}$ and $L_{3}$ varies from $2 \mathrm{~mm}$ to $8 \mathrm{~mm}$. The $S_{11}$ curves of the

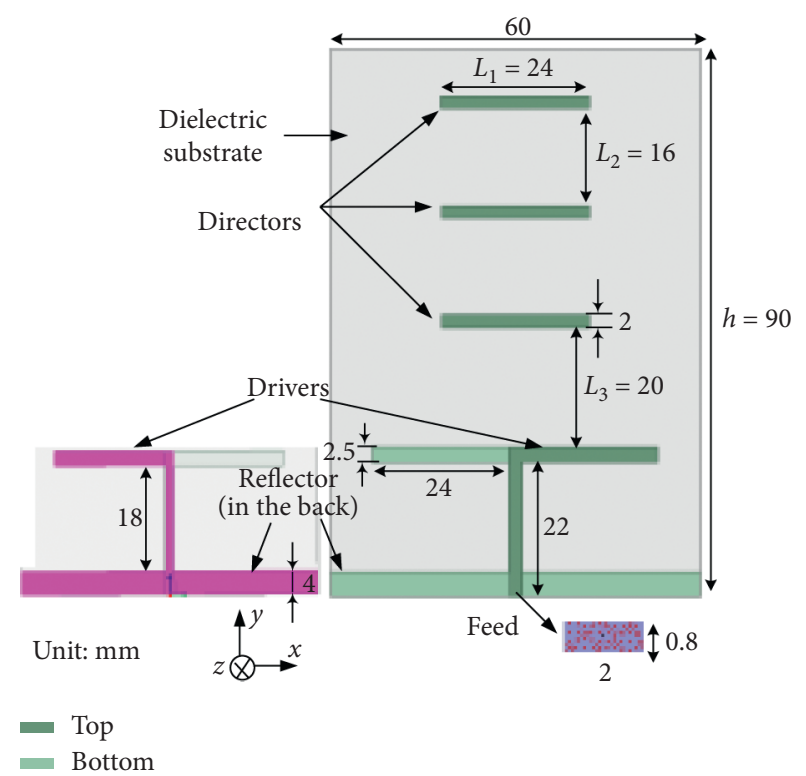

FIGURE 1: Layout and design parameters of the reference quasi-Yagi antenna with straight arms.

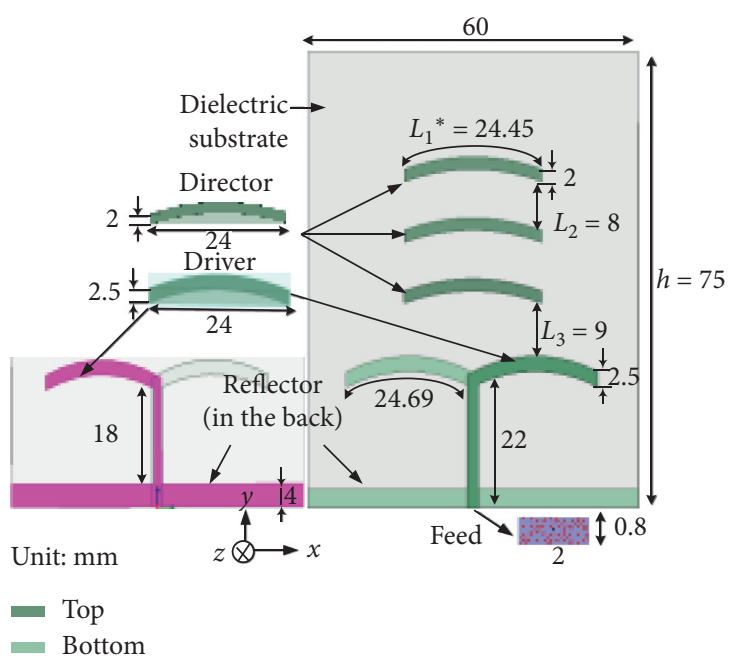

FIGURE 2: Layout and design parameters of the quasi-Yagi antenna with bent arms.

abovementioned quasi-Yagi antennas are compared and shown in Figure 5.

As clearly demonstrated in Figure 5, the frequency offset of the quasi-Yagi antenna with straight arms is more than $60 \mathrm{MHz}$ under the abovementioned parameter variation, but the frequency offset of the quasi-Yagi antenna with bent arms is less than $20 \mathrm{MHz}$. It reveals that the resonant frequency is hardly affected by structure modification of the quasi-Yagi antenna with bent arms, which is more stable than the antenna with straight arms. Moreover, the comparison results indicate the quasi-Yagi with bent arms could be further miniaturized with lower loss. 


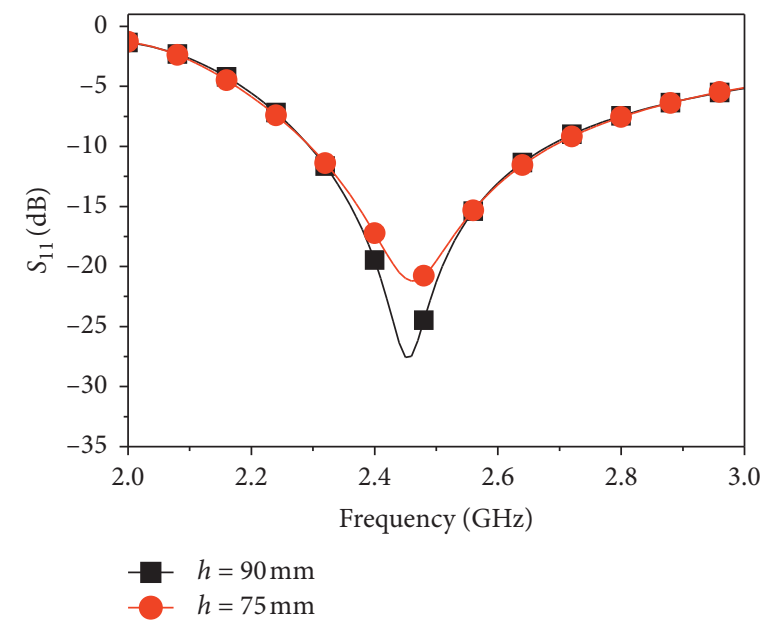

Figure 3: Comparison of the reflection coefficients.

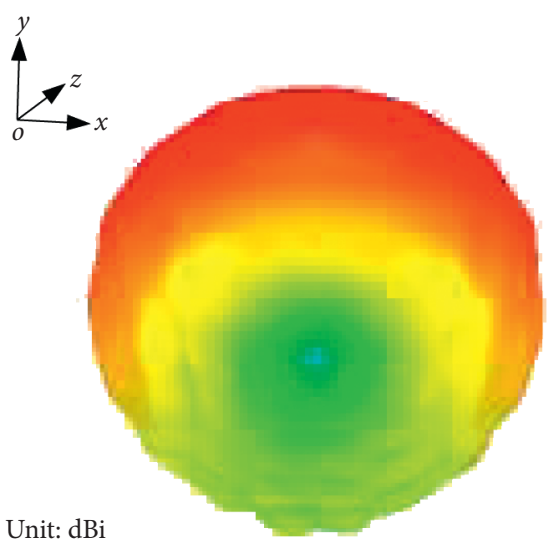

(a)

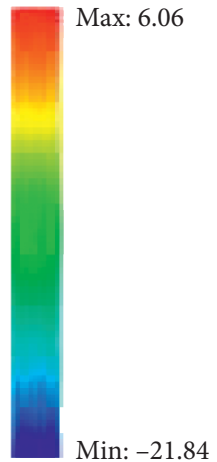

Min: -21.84

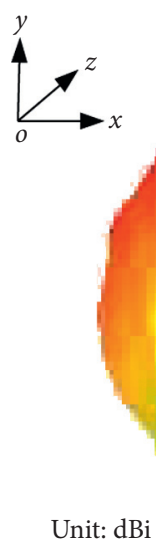

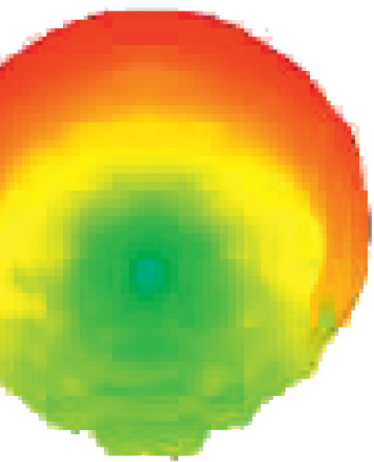

(b)

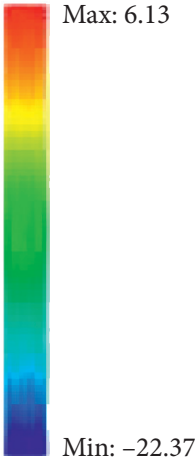

Min: -22.37

FIgURE 4: Comparison of the 3D radiation patterns. (a) Straight arms. (b) Bent arms.

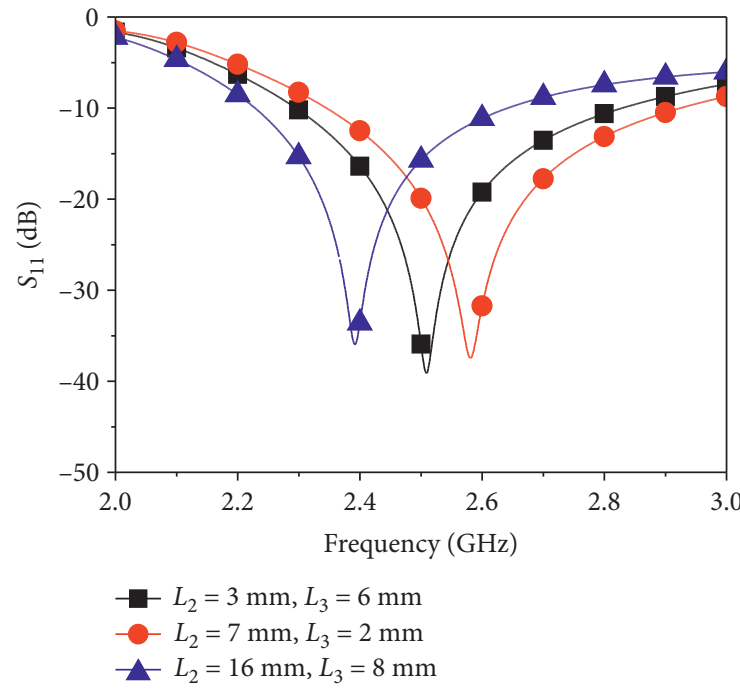

(a)

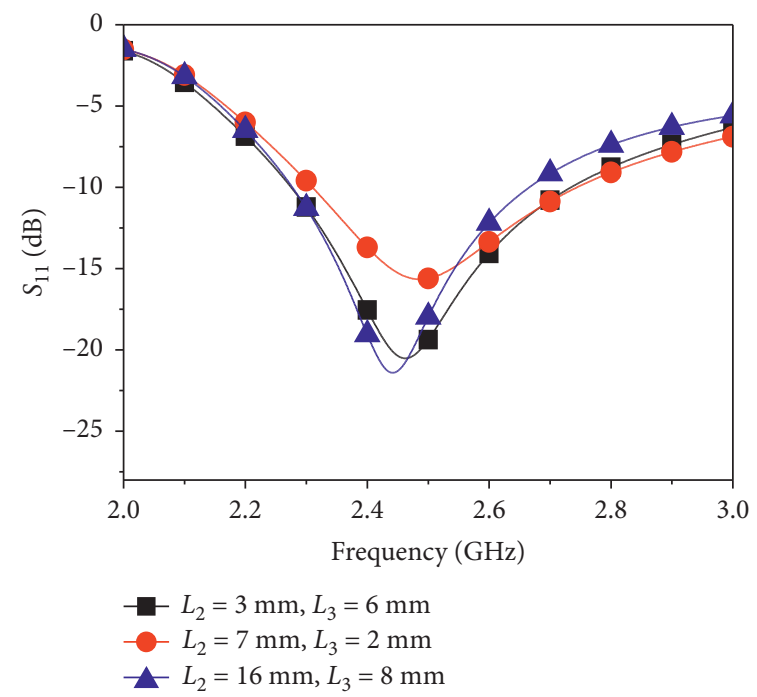

(b)

FIgURE 5: Variation of $S_{11}$ versus resonant frequency of different parameters. (a) Straight arms. (b) Bent arms. 


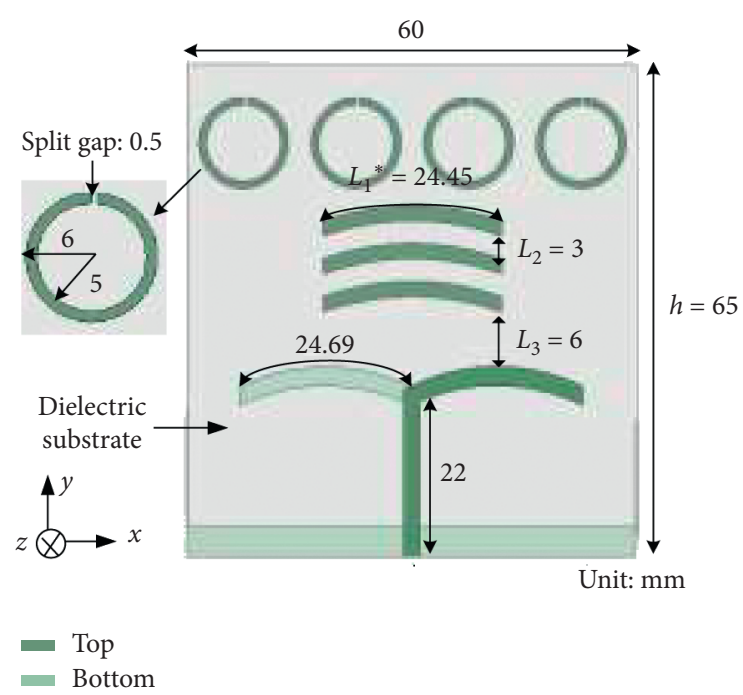

(a)

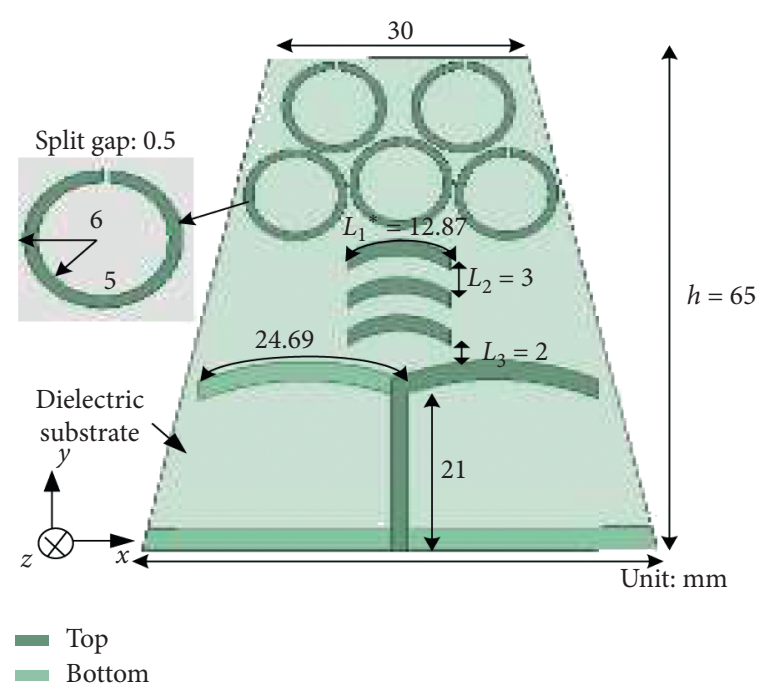

(b)

FiguRE 6: Layout and design parameters of the proposed compact quasi-Yagi antennas with bent arms. (a) Compact rectangle quasi-Yagi antenna. (b) Compact trapezoid quasi-Yagi antenna.

\section{A Compact Quasi-Yagi Antenna with SRRs}

If the size of the initial model of quasi-Yagi antenna with bent arms is further reduced, its gain would also be reduced. To overcome this problem, metamaterials were introduced into the design of Yagi-Uda antennas [19-22]. In [23], we have used SRRs to reduce coupling between antenna elements. In this article, the SRRs array is used as additional parasitic elements, which can generate the induced currents of the proposed antenna and compensate the gain reduction in its miniaturization process. Based on the simulated results in section 2, a compact rectangle quasi-Yagi antenna with SRRs is proposed firstly, as shown in Figure 6(a). A compact trapezoid quasi-Yagi antenna is then designed to further reduce the antenna size, as shown in Figure 6(b).

The reflection coefficients of the two shapes of compact quasi-Yagi antennas are simulated. As Figure 7 shows both compact quasi-Yagi antennas resonate at $2.45 \mathrm{GHz}$ with a good impedance matching level.

The gains of the compact rectangle antenna and trapezoid antenna at the operation frequency of $2.45 \mathrm{GHz}$ were also calculated, and the results are shown in Figure 8.

As seen in Figure 8, the compact rectangle quasi-Yagi antenna exhibits a maximum gain of $6.58 \mathrm{dBi}$, and that of the compact trapezoid quasi-Yagi antenna is $6.54 \mathrm{dBi}$. The simulated results indicate that the shorter bent arms of directors and more SRRs would not change the resonant frequency either.

The simulated surface current distributions of the proposed antenna are shown in Figure 9. Since the magnetic field of the proposed quasi-Yagi antenna passes through the SRR loop perpendicularly, the SRRs, which can be treated as an additional parasitic element, will resonate at $2.45 \mathrm{GHz}$ and generate in phase current distribution in the loops. As a result, it can further enhance the end-fire radiation performance due to the constructive interference in this direction. Therefore, the gain of the proposed quasi-Yagi antenna is improved without affecting the antenna size and bandwidth.

\section{Quasi-Yagi Antennas Fabrication and Their Measurement Results}

The prototype of the reference quasi-Yagi antenna and the proposed quasi-Yagi antennas is fabricated, as shown in Figure 10. The fabricated dielectric substrate has the same relative permittivity and thickness as the simulation model. The reference antenna is shown in Figure 10(a), and the proposed compact quasi-Yagi antennas with rectangle and trapezoid shape are observed in Figures 10(b) and 10(c).

The fabricated antennas were measured by using the Agilent Technologies E5071C vector network analyzer. The comparison of $S_{11}$ between the measured and simulation results are shown in Figure 11.

As can be seen clearly in Figure 11, the simulated and measured results of the reflection coefficients are in a good agreement. The measured results indicate the resonant frequency of three antennas is $2.45 \mathrm{GHz}$ with reflection coefficients better than $-18 \mathrm{~dB}$. It also reveals the ideal response of the reflection coefficients obtained by adding the SRRs.

Figure 12 shows the comparisons of simulated and measured radiation patterns in the $E$ and the $H$ plane at $2.45 \mathrm{GHz}$.

As can be seen clearly in Figure 12, the agreement between the simulated and measured patterns is satisfactory. The small difference in the back lobe radiation between simulated and measured results is mainly due to the fabricated accuracy and measurement error. Generally speaking, the performance of the main lobe suggests that the proposed quasi-Yagi antenna has good end-fire performance at the operation frequency. 


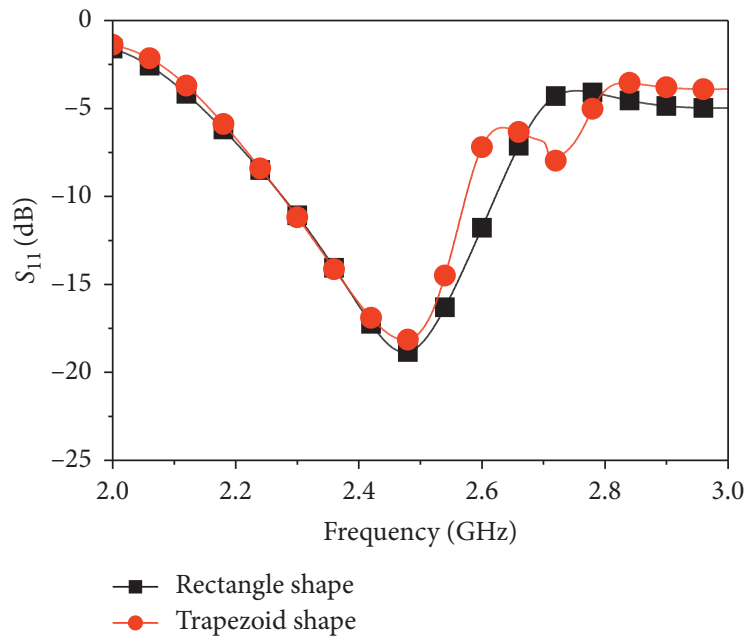

FIGURE 7: Reflection coefficients of the compact quasi-Yagi antennas.

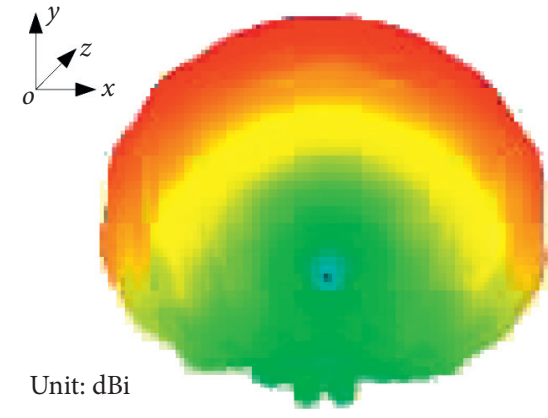

(a)

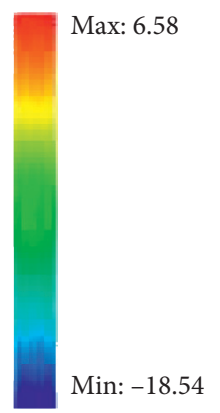

Min: $-18.54 \quad$ Unit: $\mathrm{dB}$

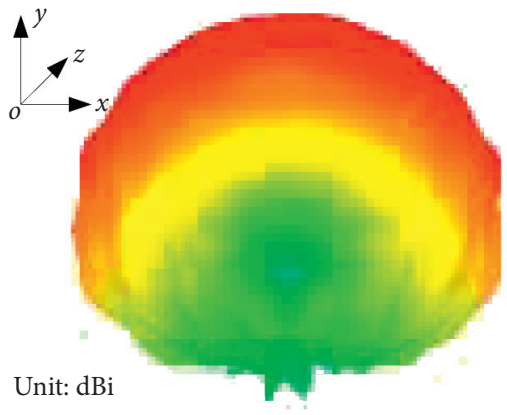

(b)

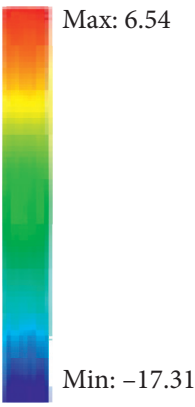

Min: -17.31

FIGURE 8: 3D radiation patterns of the compact quasi-Yagi antennas. (a) Rectangle shape. (b) Trapezoid shape.

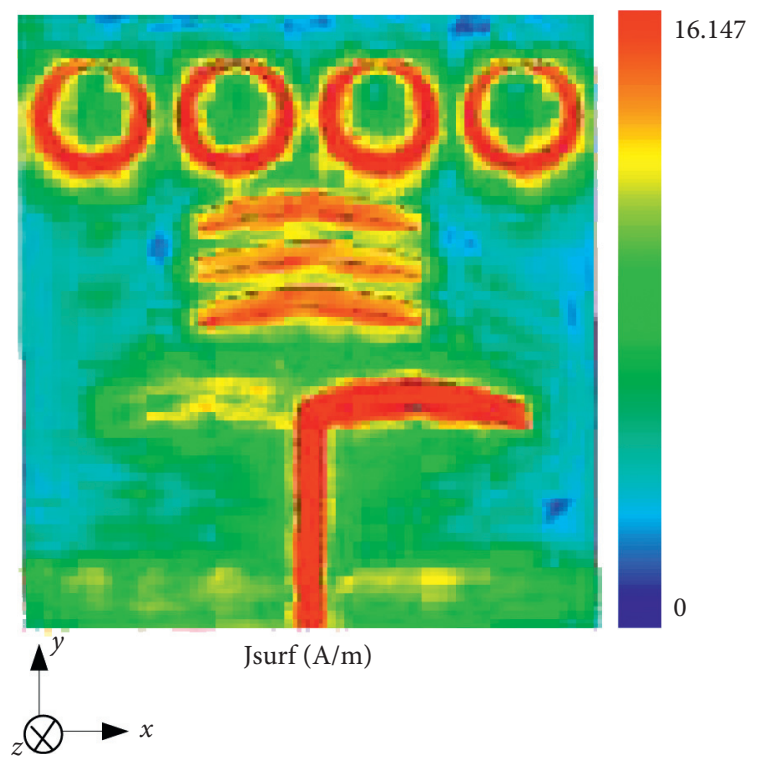

(a)

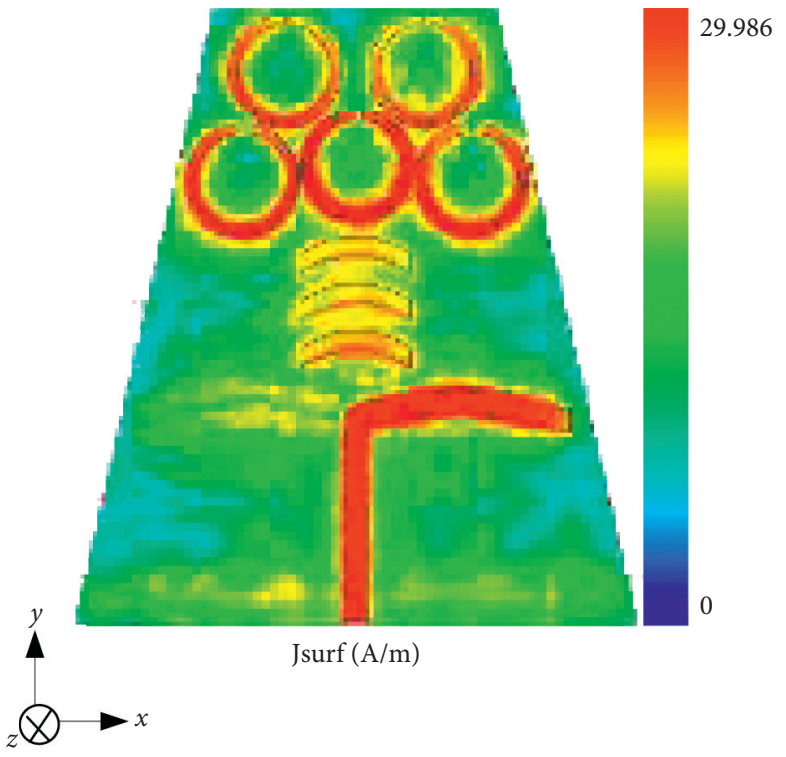

(b)

Figure 9: Simulated surface current distribution of the compact quasi-Yagi antennas. (a) Rectangle shape. (b) Trapezoid shape. 


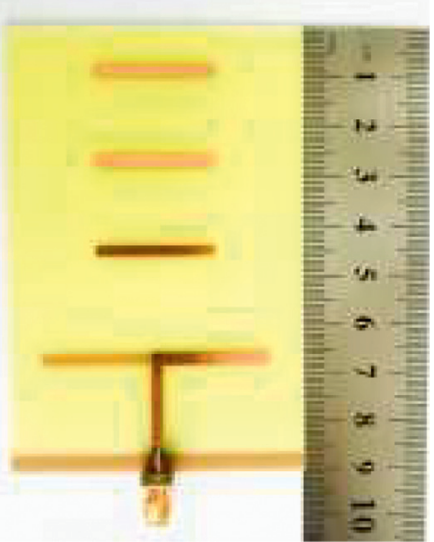

(a)

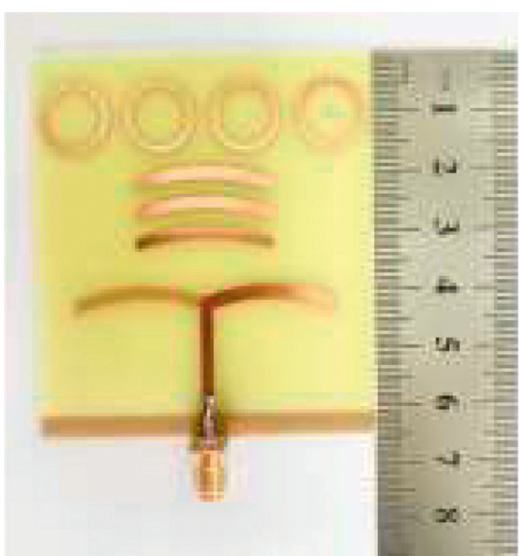

(b)

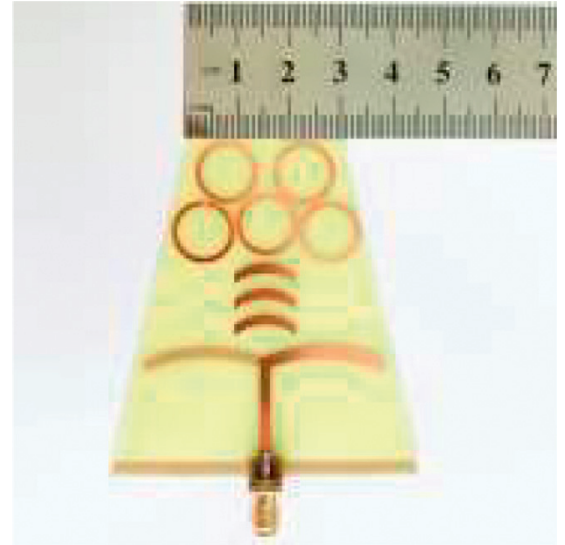

(c)

FIgURE 10: Fabricated quasi-Yagi antennas. (a) Reference quasi-Yagi antenna with straight arms (90 mm). (b) Compact rectangle quasi-Yagi antenna with bent arms $(65 \mathrm{~mm})$. (c) Compact trapezoid quasi-Yagi antenna with bent arms $(30 \mathrm{~mm})$.

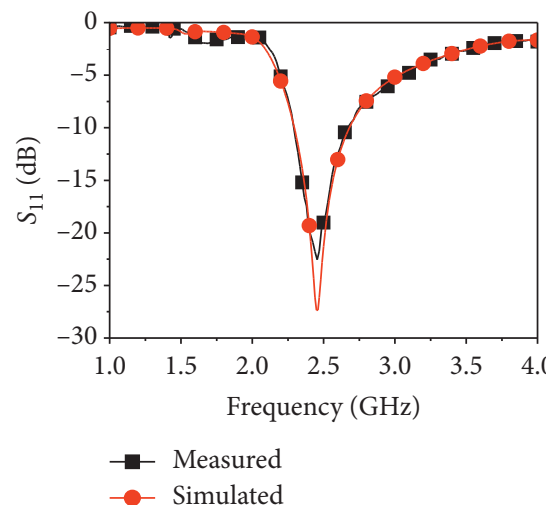

(a)

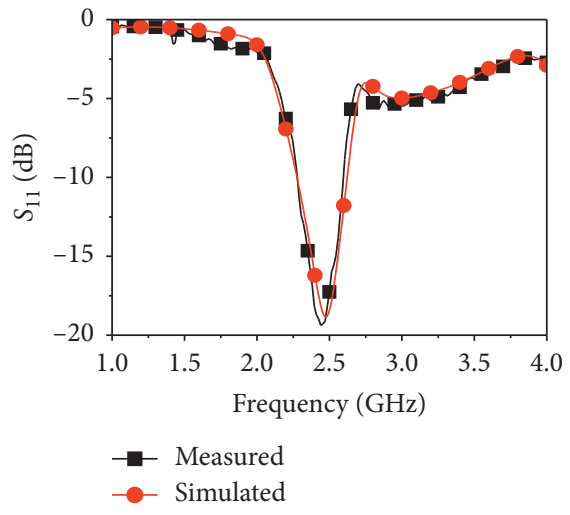

(b)

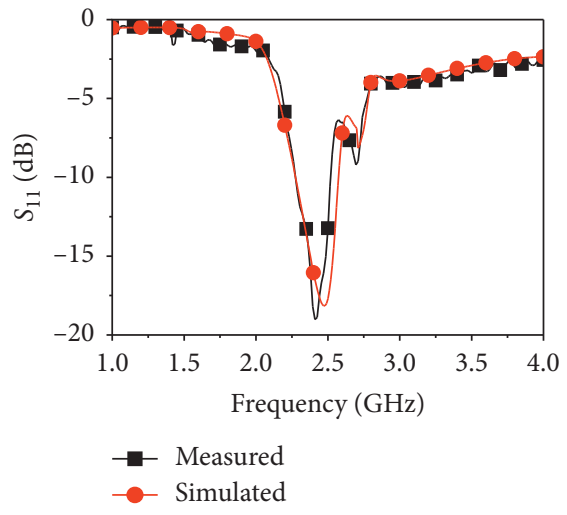

(c)

FIGURE 11: $S_{11}$ comparison of the fabricated quasi-Yagi antennas. (a) Reference quasi-Yagi antenna with straight arms (90 mm). (b) Compact rectangle quasi-Yagi antenna with bent arms $(65 \mathrm{~mm})$. (c) Compact trapezoid quasi-Yagi antenna with bent arms $(30 \mathrm{~mm})$.

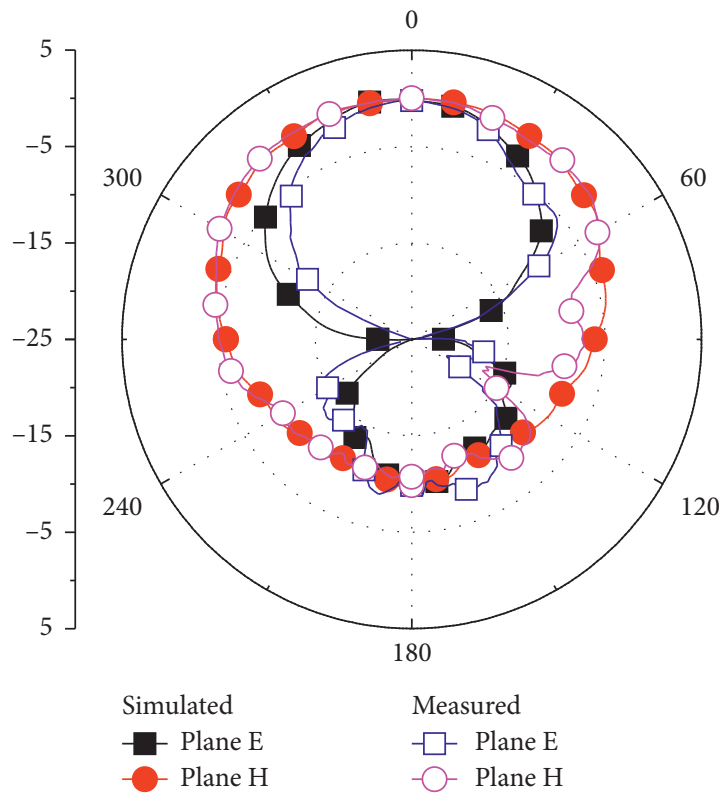

(a)

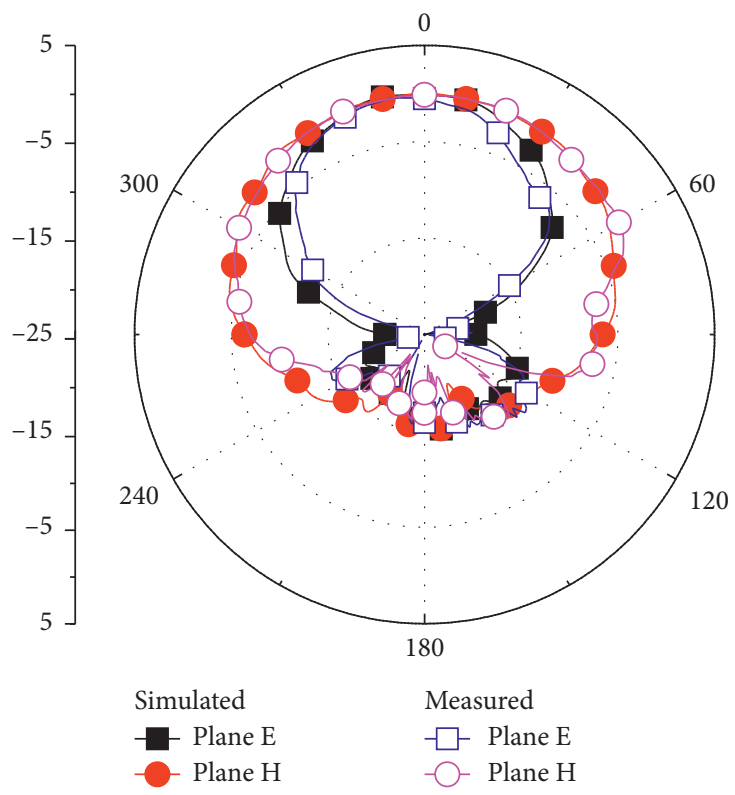

(b)

Figure 12: Continued. 


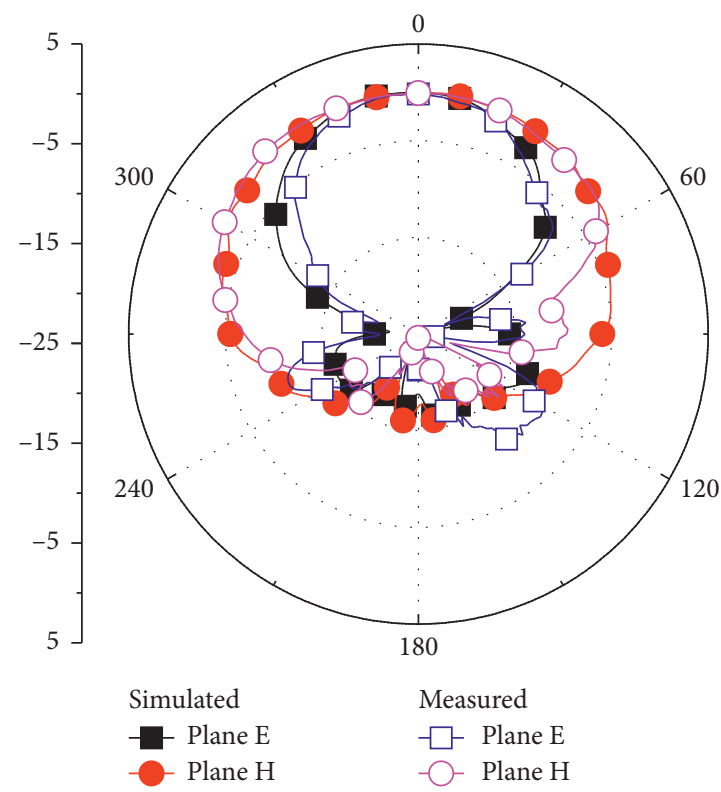

(c)

FIGURE 12: Simulated and measured radiation patterns of the designed quasi- Yagi antenna. (a) Reference quasi-Yagi antenna (90 mm). (b) Compact rectangle quasi-Yagi antenna $(65 \mathrm{~mm})$. (c) Compact trapezoid quasi-Yagi antenna $(30 \mathrm{~mm})$.

TABLe 1: Detailed comparison of the presented antennas.

\begin{tabular}{lcc}
\hline & Bandwidth/(band) (GHz) & Radiation efficiency \\
\hline Reference & $0.34(2.3 \sim 2.68)$ & 0.95418 \\
Rectangle shape & $0.35(2.285 \sim 2.635)$ & 0.95504 \\
Trapezoid shape & $0.3(2.275 \sim 2.575)$ & 0.95598 \\
\hline
\end{tabular}

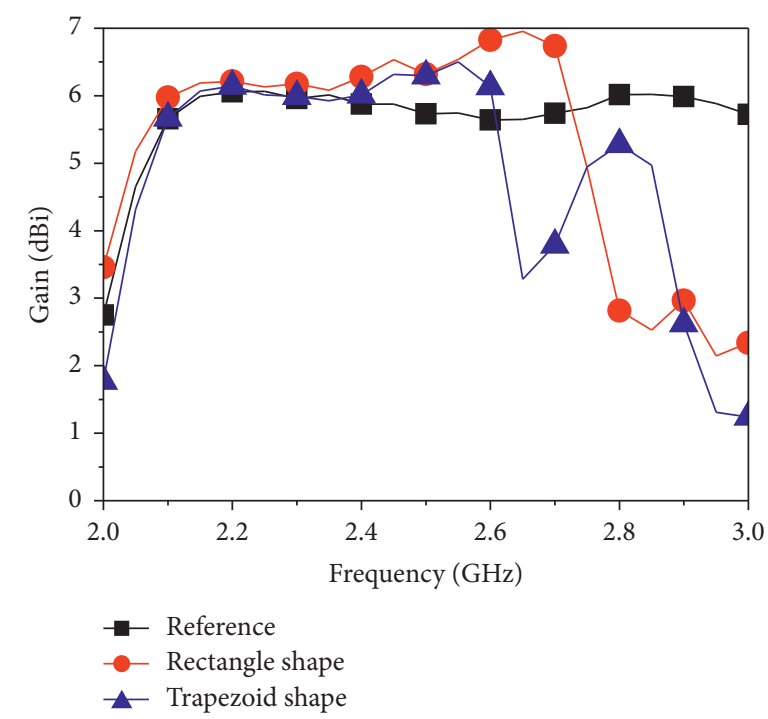

FIGURE 13: Simulated results of 2D gain versus frequency comparison of the presented antennas. 
TABLE 2: Comparison of the proposed quasi-Yagi antenna with other quasi-Yagi works.

\begin{tabular}{|c|c|c|c|c|c|c|c|c|}
\hline References & {$[10]$} & [11] & {$[12]$} & {$[13]$} & {$[14]$} & {$[15]$} & {$[16]$} & Proposed \\
\hline Frequency $(\mathrm{GHz})$ & 0.9 & 0.92 & 2 & 2.4 & 4.5 & 2.25 & 2.45 & 2.45 \\
\hline Size $\left(\mathrm{mm}^{2}\right)$ & $90 \times 90$ & $80 \times 45.5$ & $55 \times 60$ & $70 \times 49$ & $55 \times 35$ & $97 \times 86$ & $104 \times 72.5$ & $65 \times 60$ \\
\hline Gain $(\mathrm{dBi})$ & 5.3 & 4.5 & 5.36 & 5.7 & 4.6 & 5.3 & 6.5 & 6.58 \\
\hline Design complexity & Complex & Complex & Complex & Easy & Easy & Easy & Complex & Easy \\
\hline
\end{tabular}

Detailed bandwidth description versus operating frequency of the proposed antennas and their corresponding radiation efficiency at $2.45 \mathrm{GHz}$ are displayed in Table 1 .

As can be seen in Table 1, the $10 \mathrm{~dB}$ return loss bandwidth of the presented antennas are more than $0.3 \mathrm{GHz}$, and the radiation efficiency are more than 0.95 at $2.45 \mathrm{GHz}$. The compact rectangle antenna has the best performance.

To compare the gain enhancement of 3 presented antennas, the 2D gain versus frequency plot for simulation results is given, as shown in Figure 13. It can be seen that the gain of the compact antennas with SRRs is higher than that of the antenna without SRRs at their corresponding operating frequency band.

We also compare the performance of the proposed quasi-Yagi antenna with other compact Yagi works, and detailed descriptions are shown in Table 2. As shown in Table 2, the proposed antenna has the simple structure, relatively compact size, and high gain. The design complexity refers to the flexibility of parameters adjustment in the realization of Yagi antenna miniaturization.

\section{Conclusions}

In this work, a compact quasi-Yagi antenna by embedding bent arms and SRRs is proposed. The stability of resonant frequency is examined using four variable values, $L_{1 *}, L_{2}, L_{3}$, and numbers of SRRs. The antenna always keeps the same resonant frequency of $2.45 \mathrm{GHz}$ basically, which means that the proposed antenna could be flexibly miniaturized in a certain extent. The SRRs are arranged in the front of directors to enhance the end-fire gain to $6.58 \mathrm{dBi}$. The simulation and experimental results show that the geometrical size of the quasi-Yagi antenna can be reduced by $28 \%(0.21$ $\lambda_{0}$ ), with its gain increase of $8.6 \%$ by the proposed design method of combining bent arms with SRRs. The better match in impedance and the lower reflection loss can be obtained at the same time. The proposed compact quasi-Yagi antenna with high gain can be used in point-to-point wireless communication systems and the massive array antennas.

\section{Data Availability}

The data used to support the findings of this study are included within the article.

\section{Conflicts of Interest}

The authors declare that they have no conflicts of interest.

\section{Acknowledgments}

This research was financially supported by the National Natural Science Foundation of China (61701208 and 61631007), Natural Science Foundation of Gansu Province (20JR10RA604), and the Tianyou Youth Talent Lift Program of Lanzhou Jiaotong University (1520260111).

\section{References}

[1] F. Paredes, G. Z. Gonzàlez, J. Bonache, and F. J. Martin, "Dual-band impedance-matching networks based on splitring resonators for applications in RF identification (RFID)," IEEE Transactions on Microwave Theory and Techniques, vol. 58, no. 5, pp. 1159-1166, 2010.

[2] F. Sun, F.-S. Zhang, H. Zhang, H. Zhang, C. Li, and C. Feng, "A frequency diversity printed-Yagi antenna element for apertures selectivity wideband array application," IEEE Transactions on Antennas and Propagation, vol. 66, no. 10, pp. 5634-5638, 2018.

[3] S. S. Jehangir and M. S. Sharawi, "A miniaturized UWB biplanar Yagi-like MIMO antenna system," IEEE Antennas and Wireless Propagation Letters, vol. 16, pp. 2320-2323, 2017.

[4] Z. Chen, M. Zeng, A. S. Andrenko, Y. Xu, and H. Z. Tan, “A dual-band high-gain Quasi-Yagi antenna with split-ring resonators for radio frequency energy harvesting," Microwave and Optical Technology Letters, vol. 61, no. 9, pp. 2174-2181, 2019.

[5] H. Raad, "A Yagi-Uda antenna array for conformal IOT and wireless charging applications," Microwave and Optical Technology Letters, vol. 61, no. 3, pp. 633-637, 2019.

[6] L. Zheng, H. Zhai, Z. Wei, S. Ma, and J. Shi, "A new wide stopband and high gain quasi-Yagi filtering antenna," Microwave and Optical Technology Letters, vol. 61, no. 1, pp. 131-135, 2019.

[7] S. I. H. Shah, M. M. Tentzeris, and S. Lim, "A deployable quasi-Yagi monopole antenna using three origami magic spiral cubes," IEEE Antennas and Wireless Propagation Letters, vol. 18, no. 1, pp. 147-151, 2019.

[8] Z. Yang, L. Zhang, and T. Yang, "A microstrip magnetic dipole Yagi-Uda antenna employing vertical I-shaped resonators as parasitic elements," IEEE Transactions on Antennas and Propagation, vol. 66, no. 8, pp. 3910-3917, 2018.

[9] C. H. Wu, C. H. Wang, S. Y. Chen et al., "Balanced-to-Unbalanced bandpass filters and the antenna application," IEEE Transactions on Microwave Theory and Techniques, vol. 56, no. 11, pp. 474-2482, 2008.

[10] J. Yeo and J.-I. Lee, "Design of miniaturized quasi-Yagi antenna for portable RFID reader applications," Progress in Electromagnetics Research C, vol. 58, pp. 97-104, 2015.

[11] J.-H. Kim, M.-G. Jeong, S.-H. Bae, and W.-S. Lee, "Miniaturized printed YAGI-Uda antenna with high directivity for long range UHFRFID item tracking systems," Microwave and Optical Technology Letters, vol. 59, no. 2, pp. 439-441, 2017. 
[12] S. S. Jehangir and M. S. Sharawi, "A miniaturized multi-wide band Quasi-Yagi MIMO antenna system," International Journal of RF and Microwave Computer-Aided Engineering, vol. 28, p. e21237, 2018.

[13] G. Liu, Y. M. Pan, T. L. Wu, and P. F. Hu, "A compact planar quasi-Yagi antenna with bandpass filtering response," IEEE Access, vol. 7, pp. 67856-67862, 2019.

[14] K. D. Xu, H. Xu, and Y. Liu, "Low-profile filtering end-fire antenna integrated with compact bandstop filtering element for high selectivity," IEEE Access, vol. 99, pp. 1-6, 2019.

[15] A. D. Chaudhari and K. P. Ray, "Compact broadband printed Quasi-Yagi antenna with series-fed double monopole," $\mathrm{Mi}$ crowave and Optical Technology Letters, vol. 62, no. 4, pp. 1807-1814, 2020.

[16] S. Fu, A. Xiong, W. Chen, and S. Fang, "A compact planar Moxon-Yagi composite antenna with end-fire radiation for dual-band applications," Microwave and Optical Technology Letters, vol. 62, no. 6, pp. 2328-2334, 2020.

[17] S. Ahidi Rezacich, M. A. Antoniades, and A. M. Abbosh, "Gain enhancement of wideband metametial-loaded loop antenna with tightly coupled arc-shaped directors," IEEE Transactions on Antennas and Propagation, vol. 65, no. 4, pp. 2090-2095, 2017.

[18] J. Y. Mao, Z. R. Li, Q. X. Guo et al., “A wideband Quasi-Yagi antenna with arrow-shaped dipoles for digital TV band applications," Journal of Electromagnetic Waves and Applications, vol. 26, no. 13, pp. 1716-1723, 2012.

[19] M. Aeini, S. Jarchi, and R. Faraji-Dana, "Compact, widebandprinted quasi-Yagi antenna using spiral metamaterial resonators," Electronics Letters, vol. 53, no. 21, pp. 1393-1394, 2017.

[20] O. M. Khan, Z. U. Islam, Q. U. Islam, and F. A. Bhatti, "Multiband high-gain printed Yagi array using square spiral ring metamaterial structures for S-band applications," IEEE Antennas and Wireless Propagation Letters, vol. 13, pp. 1100-1103, 2014.

[21] P. Aguil'a, S. Zuffanelli, G. Zamora et al., "Planar Yagi-Uda antenna array based on split ring resonators (SRRs)," IEEE Antennas and Wireless Propagation Letters, vol. 16, pp. 1233-1236, 2017.

[22] Y.-H. Ren, J. Ding, C.-J. Guo, Y.-C. Song, and Y. Qu, “A wideband gain-enhanced dual-polarized printed antenna based on log-periodic parasitic directors (LPPDs)," Journal of Electromagnetic Waves and Applications, vol. 30, no. 8, pp. 1021-1031, 2016.

[23] W. Zhou and M. Lu, "Miniaturization of quasi-Yagi antenna array with high gain using split-ring resonators," International Journal of Antennas and Propagation, vol. 2020, Article ID 4915848, 12 pages, 2020. 